\title{
Tolerance and xenograft survival
}

\author{
David H. Sachs, Megan Sykes, Julia L. Greenstein ${ }^{1} \&$ A. Benedict Cosimi \\ Harvard Medical School and Massachusetts General Hospital, MGH East, Building 149-9019, 13th Street, \\ Boston, Massachusetts 02129, USA, and 'BioTransplant, Inc., Charlestown, Massachusetts 02129, USA \\ Correspondence should be addressed to D.H.S.
}

Hyperacute rejection (HAR) of discordant xenogeneic organs can be avoided by either removing natural antibodies (plasmapheresis and/or absorption) or by interfering with complement activation (soluble complement inhibitors or genetic engineering of the donor). However, long-term survival will also require attention to cellular pathways of rejection, which appear to be even more potent than those encountered by allotransplants. With sufficient immunosuppression it should be possible to make any xenograft survive. Nevertheless, one can predict that the intensity of immunosuppression required to overcome xenogeneic responses will result in clinically unacceptable complication rates. Induction of tolerance to xenogeneic antigens recognized in the human anti-pig cellular immune response may therefore be the key to long-term successful xenotransplantation.

\section{Mixed chimaerism and tolerance}

In this laboratory we have studied mixed haematopoietic chimaerism as an approach to allogeneic and xenogeneic transplantation tolerance. This approach involves transient ablation of the recipient's immune system, during which time partial donor haematopoietic repopulation is established by a bone marrow transplant. The immune system then reconstitutes in the presence of both recipient and donor haematopoietic elements, and becomes tolerant to both. Most important, a normal immune response to all other antigens, including pathogenic organisms, is restored.

We have previously applied this approach successfully in rodents, both for allografts $^{1}$ and for concordant (rat-tomouse) xenografts ${ }^{2}$. We have also demonstrated that similar conditioning leads to long-term survival ( $>2$ years) of kidney transplants in fully mismatched non-human primates receiving no immunosuppressive agents after the first month ${ }^{3}$. In addition, we have preliminary evidence that the same approach may be effective in a concordant (baboonto-cynomolgus monkey) xenotransplant model, in which one recipient has now survived with normal renal xenograft function for more than 120 days (J. Powelson et al., unpublished data). We are now studying the extension of this approach to the most relevant system for clinical applications, pig to primate ${ }^{4}$. The approach includes all of the elements used in the primate allograft model $^{3}$ plus absorption of natural antibodies and a two-week course of DSG.

\section{Removal of natural antibodies}

In initial studies, intraoperative perfusion of monkey blood through a pig liver removed circulating natural antibodies and prevented $\mathrm{HAR}^{4}$. We have now demonstrated that similar absorption can be accomplished by perfusion through columns of a matrix containing the $\alpha-1,3-\mathrm{Gal}$ epitope (manuscript in preparation).

\section{Xenograft survival}

In a series of 15 technically successful xenotransplants using this protocol, kidney survival times ranged from 5 to 15 days $^{s}$. Natural antibodies were not detectable in the post-transplant sera of most recipients, but rejection was accompanied by the production of induced antibodies directed toward both $\alpha-1,3-\mathrm{Gal}$ and other epitopes. These induced antibodies were mainly IgG, and presumably the result of a T-cell dependent response. Previous studies

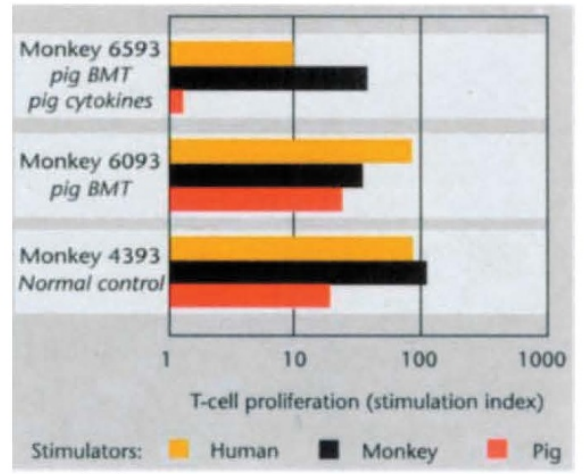

Specific loss of xeno MLR to pig stimulators in monkey 6593 , treated with pig recombinant cytokines. Monkey 6093 received the same protocol but did not receive pig cytokines (T. Sablinski et al., manuscript in preparation). in our rodent model have shown that tolerance induced by mixed chimaerism avoids such humoral responses ${ }^{6}$. Thus, although HAR was prevented, we had not induced tolerance.

\section{Bone marrow engraftment}

Our studies in rodents and non-human primates have indicated that tolerance induction by this protocol is dependent on engraftment of donor marrow ${ }^{1-3}$. However, in these pig-to-monkey studies, circulating pig cells were detected only transiently ${ }^{4,5}$. As failure of engraftment in this discordant xenogeneic system could result from the absence of pig-specific growth factors, we have produced several recombinant swine cytokines (manuscript in preparation) and begun experiments using these cytokines postoperatively. In an initial study in which pig recombinant stem-cell factor and rIL-3 were administered for two weeks after a pig bone marrow transplant, survival of pig bone marrow progeny in the monkey's bone marrow was detected for more than six months ${ }^{5}$. In addition, the monkey's mixed lymphocyte reaction to pig was lost specifically in this animal (see figure). Therefore, administration of pigspecific growth factors may provide the appropriate conditions for successful marrow engraftment and tolerance to discordant organ xenografts.

1. Sharabi, Y.\& Sachs, D.H. Mixed chimerism and permanent specific transplantation tolerance induced by a nonlethal preparative regimen. J. exp. Med. 169, 493-502 (1989).

2. Sharabi, Y., Aksentijevich, I., Sundt T.M., Sachs, D.H. \& Sykes, M. Specific tolerance induction across a xenogeneic barrier: Production of mixed rat/mouse lymphohematopoietic chimeras using a nonlethal preparative regimen. J. exp. Med. 172, 195-202 (1990).

3. Kawai, T. et al. Mixed allogeneic chimerism and renal allograft tolerance in cynomolgus monkeys. Transplantation 59, 256-262 (1995).

4. Tanaka, M. et al. Xenotransplantation from pig to cynomolgus monkey: The potential for overcoming xenograft rejection through induction of chimerism. Transplantn Proc. 26, 1326-1327 (1994).

5. Sachs, D.H. \& Sablinski, T. Tolerance across discordant xenogeneic barriers. Xenotransplantation (1995) (in the press)

6. Aksentijevich, I., Sachs, D.H. \& Sykes, M. Humoral tolerance in xenogeneic BMT recipients conditioned with a non-myeloablative regimen. Transplantation 53, 1108-1114 (1992). 